\title{
Erratum: Vascular networks due to dynamically arrested crystalline ordering of elongated cells [Phys. Rev. E 87, 012725 (2013)]
}

\author{
Margriet M. Palm and Roeland M. H. Merks
}

(Received 5 June 2013; published 18 June 2013)

DOI: 10.1103/PhysRevE.87.069903

PACS number(s): 87.17.Jj, 87.17.Pq, 83.80.Xz, 87.18.Hf, 99.10.Cd

We erroneously used a three-dimensional definition of the nematic order parameter $S(r)$ instead of the two-dimensional order parameter $S(r)=\langle\cos [2 \theta(\vec{X}(\sigma), r)]\rangle_{\sigma}$ appropriate for our model, such that for the nonordered state $S(r)=1 / 4$ instead of $S(r)=0$. In addition, we identified a small bug in our analysis scripts, which sometimes yielded incorrect values for the angles $\theta$ between the cells and the local director. Figures 1(a) and 1(b) plot the correct angles $\theta$ using the same morphologies as in Figs. 2(a) and 2(b) of the original paper, and Fig. 1(c) plots the correct values of $S(r)$. The bug slightly affected the delineation of the cell clusters used for the calculations of the translational and rotational diffusion coefficients (Fig. 2). Overall, the errors had very minor effects on our results and did not change our conclusions.

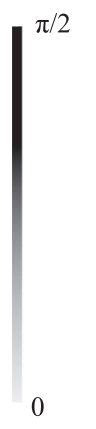

(a)

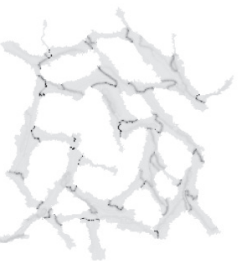

no chemotaxis

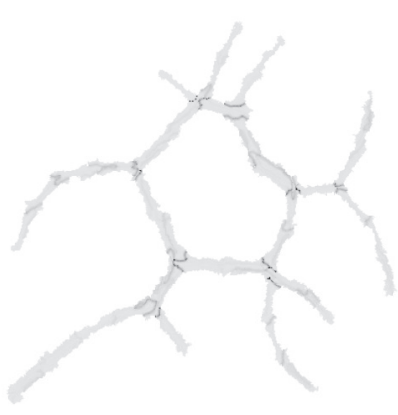

(b) chemotaxis

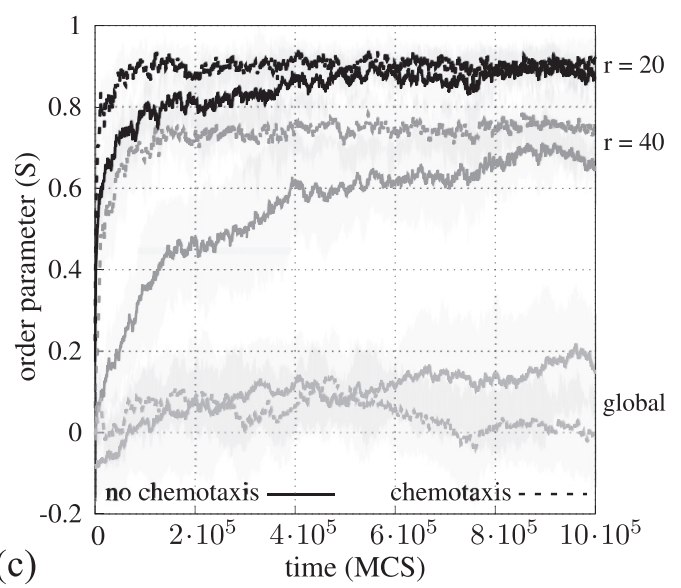

(c)

FIG. 1. Corrected Fig. 2.

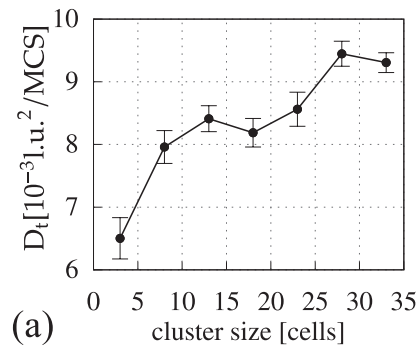

FIG. 2. Corrected Fig. 3.

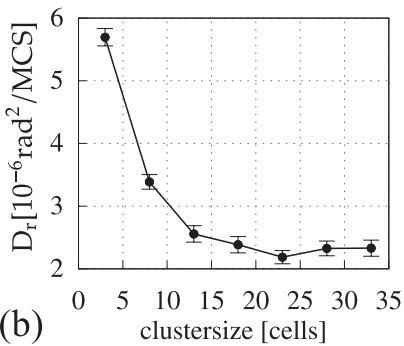

